\title{
LA DISCUSSION PACIFIQUE COMME RAPPORT À LA LANGUE FRANÇAISE SUR WIKILF
}

\author{
THE PEACEFUL DISCUSSION AS A RELATIONSHIP TO THE \\ FRENCH LANGUAGE ON THE CREATING LANGUAGE WEBSITE
}

Léda Mansour ${ }^{157}$

RÉSUMÉ: Cet article veut participer aux études sur le rapport des locuteurs à leur propre langue, ici la langue française. Alors que les études sur la langue pointent un lien "passionnel" qui prendra plusieurs formes : rapport puriste (parler sans faire des fautes), rapport affectif (les mythes de beauté, d'ordre et de clarté), rapport idéologique (défendre sa langue), il existe, ailleurs, des lieux "pacifiques" où la langue n'est pas disputée mais discutée. Nous avons choisi de travailler sur le site de création langagière WikiLf; c'est en créant un nouveau mot que l'on est le plus exposé à notre façon de voir/penser notre langue. Notre analyse se centre sur l'usage du métalangage laissant émerger un certain savoir sur la langue capable de traduire autrement le rapport à sa propre langue. Nous avons démontré qu'il existe un rapport plutôt spontané des locuteurs francophones qui semble dénué de toute passion, éloigné des postures normatives. Les wikistes donnent l'exemple d'une communauté qui pacifiquement met en discussion la langue française, ce qui représente d'abord un objet de réflexion, mais encore un choix de recherche permettant de nous positionner afin de rendre compte (prendre en compte) d'une relation plus souple des usagers de la langue française.

Mots-clefs: Rapport à sa langue. Lien passionnel. Lien pacifique.

ABSTRACT: This article wants to participate in the studies on the relationship of the speakers to their own language - in this case, the French language. While the studies on the language show a "passionate" attachment that will take several forms, such as purist relationship (speaking without making mistakes), emotional relationship (the myths of beauty, order and clarity), ideological relationship (defending one's own language), there are also "peaceful" places where the language is not disputed but discussed. We chose to work on the language creating website WikiLf - it is in creating a new word that we are most exposed to the way we see / think our language. Our analysis focuses on the use of meta-language which lets some knowledge of the language to emerge, which is capable to translate the relationship to one's own language in a different way. We have demonstrated that there is a rather spontaneous relationship of Francophone speakers that seems stripped of all passion, distant from normative postures. The wikistes (the WikiLf users) give the example of a community that peacefully opens a

157 CEDITEC-Paris Est Créteil. E-mail: leda.mansour@u-paris10.fr 
discussion on the French language, which represents firstly an object of reflection, but also a research choice which allows us to position us to reflect on (and to consider) a more flexible relationship of the users of French language.

Keywords: Report to one's own language. Passionate attachment. Peaceful attachment.

\section{INTRODUCTION}

L'objectif de cet article est d'analyser le métalangage des usagers du WikiLF, un wiki d' "enrichissement de la langue française158". Nous proposons ici l'analyse qualitative de discussions observées depuis mars 2011 jusqu'à octobre 2012. Il est question de rendre compte de la complexité de ces discussions, où sont énoncées à la fois des règles de création néologique et des oppositions à ces règles, et de dégager des tendances générales sur les représentations de la langue dans un contexte de création néologique. Ce sont ces tendances générales qui construiront dans cet article ce que nous pouvons désigner par "le rapport à la langue" et permettront de le définir.

Nous décrirons les tendances qui forment les règles de création néologique suggérées par les contributeurs, ainsi que le type de métalangage mis en circulation sur WikiLF. Nous prêterons également une attention aux rares polémiques autour du vocabulaire spécialisé, ou le jargon, des linguistes.

\section{WIKILF: CORPUS, MÉTHODOLOGIE ET HYPOTHÈSES}

WikiLF est le site créé par la Délégation Générale à la Langue Française et aux Langues de France pour participer à "l'enrichissement de la langue française". Cette initiative est définie comme le fruit d'une collaboration entre des instances officielles représentées, entre autres, par la Commission générale de terminologie et de néologie, et un processus non officiel qui est celui de "l'usage que chacun en [la langue] fait":

Un néologisme ne saurait être adopté que s'il fait mouche auprès de ses potentiels usagers. C'est la raison pour laquelle le ministère de la Culture et de la Communication lance ce nouveau site collaboratif [...] : pour que ces deux processus d'enrichissement de la langue française, l'un officiel et l'autre plus diffus, prennent toute leur force en se nourrissant l'un de l'autre (http://wikilf.culture.fr/a-propos).

Les wikistes proposent des équivalents en français aux mots en anglais, ils commentent toute proposition d'un nouveau mot, adhèrent au mot créé ou le contestent. Les mots à franciser relèvent souvent du domaine informatique et technologique, mais aussi des domaines de la mode et de l'art.

Ce contexte de création néologique pourrait laisser surgir des controverses autour de la langue - ce qu'il est habituel de repérer sur les forums en ligne de l'usage de la langue (grammaire, orthographe, vocabulaire), comme le signalent des études sur la norme et sur le purisme des usagers dits communément profanes (voir entre autres Rosier 2004 et Dietmar 2004). Les recherches sur la normativité liée aux usages de

158 http://wikilf.culture.fr/ 
la langue française pointent un caractère "passionnel" qui lui serait rattaché (Rosier \& Paveau 2008). Dietmar, dans une étude sur les pratiques des internautes, parle du discours normatif comme étant "moins marqué par une approche fonctionnelle que par des considérations fort émotionnelles" (2004 : en ligne). Ce rapport "émotionnel" évoquerait des mythes rattachés à la langue française : le mythe des origines, de l'ordre logique des mots et de l'amour pour la langue, que les travaux de Meschonnic (1997) et de Cerquiglini (2007) mettent en évidence.

Sur WikiLF, à notre sens, la langue française serait plus discutée que disputée, au sens où ce site représenterait un espace de discussion d'abord orienté vers l'objectif d'enrichir la langue, bien que des objets de polémique puissent s'y manifester ; or, ce sont précisément des polémiques autour des notions spécialisées des linguistes qui apparaissent, comme nous le verrons. Ces polémiques dirigées contre les linguistes permettraient de considérer les contributeurs du WikiLF comme des non linguistes. En effet, le métalangage utilisé par les wikistes relève plutôt d'un vocabulaire technique à caractère scolaire, comme "adjectif" utilisé seul, par exemple - là où les linguistes ajouteraient sans doute des précisions supplémentaires pour éviter des usages ambigus. L'usage de notions linguistiques plus spécialisées comme "dérivation morphologique" et "idiolecte" reflète une bonne culture grammaticale chez certains wikistes, sans qu'ils recourent toutefois à une explication scientifique fort spécialisée.

Si la présence moindre de la polémique permet bien de considérer WikiLF comme un espace de discussion plutôt "pacifique", il est important néanmoins de préciser qu'au long de ces discussions, des tendances parfois opposées apparaissent dans la façon de créer un nouveau mot. Les contributeurs font de temps à autre des rappels des objectifs du site et ils se corrigent sans visée axiologique. Que signifie donc ce "rapport à la langue" dans le contexte du WikiLF?

L'analyse du métalangage utilisé par les wikistes permet de mettre en lumière leurs manières de discuter la langue française, leurs règles de création néologique et permet de décrire leur "rapport à la langue". Ce rapport, comme nous allons le montrer, semble dépourvu d'attachement nostalgique au sens que Cerquiglini donne au purisme français:

Il y a dans la langue française comme un manque. Le paradis de la latinité a été perdu; de cette perte on reste inconsolable. Une origine longtemps obscure, qui s'est finalement éclaircie dans la déception (le latin des rues comme ancêtre), une créolisation longue et inavouable (la germanisation) ont placé le français dans une relation frustrée avec la langue de prestige, le latin classique des écoles et des savants. Cela explique sans doute que le purisme, cette forme supérieure de la nostalgie, soit en français si fort et si présent (2007, p. 24).

Il apparaît que les wikistes entretiennent un lien "souple" à la langue qui les engage plutôt à répondre à des questions de type "comment enrichir la langue ?" ou encore "de quelle manière discuter de la langue ?".

Nous développons par la suite les règles de création néologique plus ou moins opposées avant de passer à la description des tendances plus communes aux contributeurs. Les deux amènent à une meilleure compréhension du rapport à la langue compris à la fois comme un moyen de créer la langue et une manière d'en parler.

L'analyse du métalangage, ici menée, vise à rendre compte des fonctions des différents usages métalinguistiques. En quoi la mobilisation du vocabulaire grammatical 
et linguistique répond-elle à certaines contraintes de création ou de représentations sur la langue ? En quoi le recours à certaines notions ou catégories servirait-elle à justifier et/ou à contester une règle de création néologique ? Notre analyse est surtout concentrée sur le contexte ou le contexte proche des termes tout en dégageant leurs éventuelles interprétations.

Elle est également qualitative qui opte pour l'étude de la comparaison du métalangage, qu'il soit fréquent et/ou peu ordinaire en construisant un corpus en termes d'objet linguistique permettant, moins d'arrêter des interprétations, que de poser des interrogations, et probablement d'en extraire des hypothèses non définitives. Nous avons donc fait le choix de travailler le contexte du néologisme car il nous a semblé un terrain fertile pour l'expression du rapport à sa propre langue, ici française ; c'est en créant un nouveau mot que l'on est le plus exposé à notre façon de voir/penser notre langue. Et nous avons opté pour l'analyse du métalangage car, en effet, ce dernier représente d'abord un outil de création néologique et ensuite laisse émerger un certain savoir sur la langue capable de traduire autrement le rapport du locuteur à sa propre langue.

Notre réflexion sur la discussion autour du discours normatif/puriste et/ou passionnel/polémique comme lien affectif à la langue française voudrait ajouter une modeste contribution afin de rendre manifeste de nouveaux liens et ce à travers la dissémination sur Wikilf d'un discours contre-normatif, pacifique, discours ici entendu au sens des travaux de Michel Foucault, à savoir une construction fragmentaire et diffuse d'un discours en lien avec une construction d'une supposée solide et unificatrice vision du monde et/ou un mode de penser/vivre (Archéologie du savoir, 1969). Comment à travers des éléments dispersés qui sont les différents usages du métalangage se forme et se dessine un certain rapport à sa langue? Nous visons donc essentiellement l'étude des effets des usages du métalangage; effet forgeant un certain discours, ici, en marge des opinions normatives sur la langue.

Notre méthodologie prend en compte l'intégralité du commentaire des internautes où apparaissent les notions, et étend l'analyse à deux, voire à trois commentaires successifs afin de mieux comprendre les fonctions et usages du métalangage. Aussi, le corpus peut-il comporter des commentaires incluant une expression ou un terme approchant une notion linguistique - l'imprécision notionnelle faisant également partie de l'analyse.

Au niveau technique, nous avons gardé l'orthographe d'origine des commentaires, en plus des pseudonymes des locuteurs-wikistes, en vertu du caractère public des commentaires et du site.

\section{RÈGLES DE CRÉATION NÉOLOGIQUE : TENDANCES OPPOSÉES}

L'usage des notions linguistiques par les wikistes montre des orientations parfois divergentes dans les règles de création. Par exemple, les propositions de traduction de "E-Reader" en français fait émerger une discussion sur le genre grammatical, une dimension qui n'est pas systématiquement prise en compte dans les règles de création revendiquées par les wikistes: ${ }^{159}$

159 À la fin des exemples, nous ajoutons le pseudonyme des wikistes, la date de publication et le terme en anglais à traduire. Nous soulignons les notions en caractères gras et les éléments nécessaires à l'analyse en caractères italiques. 
1. Pour ma première participation à ce wiki, j'aimerais proposer le terme "lisèle", venant de liseuse et électronique. Je l'imagine de genre féminin (une lisèle) (rutrapio 26 septembre 2011: E-reader)

2. Bien trouvé. Masculin ou féminin? Sachant que les deux mots-racines, "livre" et "magazine", sont masculins, mais que la finale en "ine" a une consonance plutôt féminine. Je préférais le féminin, qui prête moins à confusion (Anoup 24 janvier 2012 : Mook)

3. Merci pour avoir attiré mon attention au sujet du genre des nouveaux mots - je n'y avais jamais pensé! (thomas 24 janvier 2012 : Mook)

Ainsi, les contributeurs ne semblent pas toujours en accord sur les règles de création, ce qui est montré dans les rappels que font certains usagers.

À propos de "Peanuts" :

4. La traduction littérale et figurée est "cacah(o)uète". On dit parfaitement "C'est des cacahuètes!". Pas besoin de nouveaux mots. (LeCygne 8 octobre 2012 : Peanuts)

Cette idée sera contestée par des utilisateurs, rappelant l'objectif du site tout en utilisant la notion de "périphrase" qui serait à éviter.

À propos de "Challenging":

5. Je suis d'accord avec MissTabs. Il me semble que le but de wikiLF n'est pas de trouver dans le français les mots ou périphrases susceptibles d'être nouvellement associés à des termes étrangers, mais d'enrichir la langue française en créant. (abberwock 27 octobre 2011: challenging)

L'exemple suivant met en jeu le même type de remarques, en utilisant les notions d' "adjective" et de "substantive":

6. Mais je pense que le but serait de créer un nouveau mot, pour "enrichir" la langue française, plutôt que de recycler des mots ou périphrases existants. Qu'en pensez-vous ? J'avais initialement pensé à des adjectifs en proposant de les substantiver. (Jabberwock 27 octobre 2011: challenging)

Ces rappels vont aborder les contraintes de "concision" et d' "euphonie". À propos de "Green washing", un contributeur s'exclame:

7. écobadigeonnage me paraît un exemple de mauvais équivalent: on intellectualise, on ne s'attache qu'à la signification en oubliant les critères déterminants que sont l'euphonie, la concision, la simplicité, le potentiel réel d'utilisation. (Ehj 4 janvier 2012: Green washing)

La question du registre de langue fait aussi l'objet de discussions. Le registre "soutenu" a tendance à être écarté, comme dans la traduction du mot "liker " par "plussoyer":

8. "Pour ma part, je n'ai presque jamais utilisé "plussoyer" autrement qu'avec des camarades de promo ou encore avec d'autres mensans, donc je n'en ai jamais eu une autre impression que celle d'un terme soutenu" (Daru 3 octobre 2011 : Liker)

9. “Plussoyer est trop soutenu. Le tout est de trouver un mot qui ne fait pas partie du français soutenu, pour qu'il soit plébiscité" (Daru 23 septembre 2011 : Liker)

Si le registre soutenu ne fait pas l'unanimité, le familier semble aussi une option à écarter :

10. "Noob, ou newb sont très familiers. Il existe dans un registre de langue très proche le terme " bleu " qui correspond tout à fait" (nOOn 27 septembre 2011 : Noob ou newb)

L'usage de l'argot n'est guère favorisé: 
11. "Je propose le mot "suralcoolisme" qui n'est ni argotique ni trop familier et qui garde l'idée d'excès" (Varlet 9 mars 2012 : Binge drinking)

Si l'argot tombe dans le discrédit, le verlan fait quant à lui parti des propositions:

12. "Un fou de technologie, en verlan inversé, est un tech-ouf, à franciser en tékouf ?" (Nikos 17 octobre 2011 : Geek)

Cette relative divergence concernant le registre cède la place à un accord dès qu'il est question de traduire un nom propre. Cette dernière notion est convoquée, à plusieurs reprises afin de limiter la création néologique, comme dans les commentaires suivants à propos de "Bit torrent":

13. "BitTorrent" est un nom propre, et à part de rares exceptions, on ne traduit pas un nom propre. Bit Torrent (Motutiniad 4 octobre 2011 : Bit Torrent)

14. Tout à fait d'accord. Les mots "leech(er)" et "seed(er)" nécessitent bel et bien une traduction, mais “BitTorrent" non. C'est un nom propre, tout comme le nom d'un protocole (naixn 5 octobre 2011 : Bit Torrent)

Ainsi, les rappels de règles (nécessité d'attribuer un genre à un nom, élimination des périphrases, recherche du registre "courant", non traduction du nom propre) démontrent que les utilisateurs discutent de la langue, de ce que l'on doit faire et de ce qu'on ne peut pas faire. Le degré de divergence n'est pas cependant très élevé sur ce wiki. Nous allons à présent montrer des tendances plutôt communes aux wikistes, et qui rendent manifeste un rapport à la langue fondé sur la récupération du sens étymologique, sur le critère quantitatif, et sur un attachement à un usage des mots lié à leur contexte.

\section{RÈGLES DE CRÉATION NÉOLOGIQUE: TENDANCES COMMUNES}

Dans cette deuxième partie, nous développons les tendances fréquentes chez les wikistes, ce qui permet de définir leur rapport à la langue. Il s'agit d'un discours plutôt non puriste, sans être toutefois dénué de l'expression permanente d'un retour aux racines (latines) des mots.

\section{RECOURS À L'ÉTYMOLOGIE}

L'étymologie occupe une place considérable dans le processus créatif sur WikiLF. Les contributeurs justifient souvent leurs néologismes par une appartenance à une racine latine. Dans ce qui suit, nous prenons en compte des mots qui sont rattachés à l'étymologie comme "origine", "racine", "genèse". Ce sont ces mêmes termes qui constituent les titres des chapitres d'un livre de Cerquiglini sur le rapport du français à la filiation gréco-latine : "La fabrique de l'origine", "Les récits de la genèse" (2007).

L'exemple suivant montre comment le recours à l'étymologie constitue une étape importante chez le locuteur tamdegledel:

15. "Moi, je serais assez partant pour revenir vers l'étymologie"

Par ailleurs, Emeric75 estime que dans un néologisme l'étymologie constitue une justification de création :

16. "Pour ma part, je proposerai plutôt le néologisme "fratrien", qui se comprend sans même qu'on l'explique, et dont l'étymologie totalement limpide de "membre d'une fratrie" justifie l'emploi" (26 septembre 2011 : Sibling) 
D’autres locuteurs évoquent la "genèse des expressions" comme Pierrick déclarant:

17. "ne connaissant pas la genèse de ces expressions, dans le premier cas je peux supposer qu'il s'agit d'un véhicule domestique" (12 octobre 2011 : Break ou station wagon).

Ailleurs, eanjean préfère utiliser un terme dont la source étymologique est plus "perceptible":

18. "En langue française, la racine latine " nexus" (à l'instar de "nectere" ) est usuellement associée au préfix d'origine latine "co" (con) pour former une famille de mots allant de "connexion" à " connexité" . [...] "Connexialisme" me semble plus français et n'amoindrit ni ne déforme le sens initial ; en tout cas son étymologie est plus perceptible" (27 septembre 2011 : Nexalism)

Par ailleurs, l'expression de ce recours s'exprime en termes de "fidélité à l'esprit" dans une association de la notion de "néologisme" avec le terme de "famille":

19. “Pour l'aspect "vedete" un néologisme de la famille de notoriété/notoire pourrait convenir afin de souligner l'aspect renommée de l'invité, de la vedette ou de la personne. Par exemple "notoriant": "concert hommage à ABBA notoriant Madonna" [...] Je sais cela choque quelque peu mais je pense que cela reste fidèle à l'esprit ?!? (cyril roux 23 septembre 2011: Featuring)

Cette association peut montrer le recours à l'ancien dans le nouveau dans le contexte de création néologique, ce que Steuckardt montre dans son étude Néologie et le sentiment de la langue française au XVIIle siècle (2012). L'auteure précise: “Dans la manière de former les néologismes, une continuité demeure : ils doivent se conformer à des modèles existants" (ibid. p. 101). Le cas des contributeurs du WikiLF prouve également l'attachement au sens étymologique et l'analogie avec la langue latine. Cet attachement semble mobilisé pour créer un nouveau mot, sans que la discussion n'aille dans le sens d'une revendication d'appartenance du français à telle ou telle origine. Il semblerait que l'étymologie, bien qu'elle soit nécessaire, demeure sur WikiLF un outil de création plus qu'un objet de dispute, ce qui placerait ce recours aux racines latines à un niveau finalement méthodologique.

\section{LA NOTION DE CONTEXT}

Un des aspects sur lesquels les contributeurs du WikiLF discutent leurs néologismes est le contexte d'utilisation des mots créés. Le contexte semble poser problème aux locuteurs dans la mesure où les termes en anglais peuvent avoir plusieurs sens selon la situation d'énonciation. Par ailleurs, la notion de contexte est utilisée par les linguistes dans plusieurs domaines, ce qui n'empêcherait pas son caractère flou, voire "introuvable" comme le reformule Paveau, lors d'une conférence sur la notion de "context" (2013).

Sur WikiLF, les utilisateurs parlent souvent de la condition d'emploi. Par exemple, pour traduire "featuring", le contributeur précise les différents contextes d'usage du mot à traduire avant de créer un nouveau mot:

20. “Ce terme peut aussi bien s'employer dans le contexte d'un concert que d'un film, d'un clip ou autres représentations artistiques avec une vedette invitée-même si ce n'est pas le seul usage qu'on en fait" (Daru 9 octobre 2011 : Featuring)

Ce qui est confirmé par un autre contributeur: 
21. "Je suis d'accord que le terme "featuring" s'applique à de nombreux domaines et qu'il est nécessaire de l'adapter en fonction de son emploi" (Doni 27 septembre 2011 : Featuring)

Dans l'exemple qui suit, le contexte est lié à la notion de "polysémie":

22. "Vous avez raison nOOn. Cependant, n'oublions pas qu'en français, comme dans d'autres idiomes, de nombreux termes sont polysémiques. Ou, pour le dire autrement, leur acception peut subtilement varier de cas en cas. C'est donc en fonction du contexte qu'il faut comprendre tel mot, telle expression" (Kayowa 28 septembre 2011 : Featuring)

Dans le processus de création, la nécessité de comprendre le contexte d'usage des termes à créer est une des règles discutées par les utilisateurs, mettant au jour l'importance de la situation d'énonciation de tout néologisme; il ne suffirait pas de créer un mot, il convient avant tout de savoir l'utiliser.

\section{LE QUANTITATIF ET LA FRÉQUENCE DES MOTS}

Les contributeurs du WikiLF accordent une importance à l'argument quantitatif dans la création néologique. À cet effet, ils utilisent des termes techniques utilisés par les linguistes, comme "occurrence" . En linguistique, mesurer la fréquence et la régularité d'un terme fait partie des méthodes d'analyse des corpus. Sur WikiLF et afin de légitimer un mot créé, les contributeurs ont tendance à effectuer une recherche en ligne. Par exemple, Laurence M. cite ses sources de recherche qui sont ici l'encyclopédie collective "Wikipédia" et le moteur de recherche en ligne "Google":

23. "hashtag" = "mot-clic". Dans l'article sur "Twitter" de Wikipedia, je vois qu'on a utilisé "mot-clic" comme la traduction en français de "hashtag". J'ai vérifié "mot-clic" dans Google, et j'ai trouvé déjà 22500000 occurrences! (Laurence M. 12 mars 2012: Hashtag)

Roze utilise le qualificatif "frequent" afin de justifier le néologisme "plussoyer" pour le terme en anglais "liker":

24. "plussoyer commence à être fréquent, sympa comme néologisme" (roze 26 octobre 2011: Liker)

Ailleurs, il n'est pas question de vocabulaire technique mais de présenter un chiffre qui atteste de "l'ampleur d'utilisation" d'un mot:

25. "Et une petite recherche du genre "site:hardware.fr plussoie" dans google montre l'ampleur de son utilisation! (32000 résultats rien que pour un seul site!)" (AdriZ 3 octobre 2011 : Liker)

Nous voyons ici un certain rapport quantitatif à la langue suggérant que la quantité légitime le mot créé. L'utilisation quotidienne d'un mot, nous l'avons précisé plus haut, est le point de départ de ce wiki quand ses créateurs déclarent: "Un néologisme ne saurait être adopté que s'il fait mouche auprès de ses potentiels usagers". Or, c'est bien cet usage qui est présenté par un contributeur comme argument contre, précisément, le métalangage et le savoir spécialisé des linguistes, dans une des rares polémiques sur WikiLF :

26. "Qu'on le veuille ou non, "thriller" fait partie de notre vocabulaire quotidien depuis longtemps et est largement utilisé. Il sera donc très difficile de le remplacer. On aura beau se baser sur des études lexicales ou linguistiques pour inventer un autre mot ("frilar"), l'usage populaire ne l'adoptera pas. [...] je dirai que la 
langue est une affaire trop sérieuse pour être abandonnée aux seuls pseudolinguistes, trop enfermés dans leurs raisonnements catégoristes et qu'elle est d'abord ce que les gens en font dans leur quotidien (Varlet 3 mai 2012 : thriller)

"Notre vocabulaire quotidien", "l'usage populaire" et "ce que les gens en font dans leur quotidien" relèvent d'arguments d'adoption de tel ou tel terme en opposition à des "études lexicales ou linguistiques" et aux "raisonnements catégoristes" des "pseudo-linguistes". Cette contribution met automatiquement en opposition deux mondes de savoir sur la langue, le monde des usagers de la langue appelé "populaire" contre un monde des linguistes "enfermés" dans des catégorisations. Cette distinction est prolongée par le même contributeur:

27. "Ce site n'est pas réservé aux seuls "pros" de la linguistique. Même s'ils peuvent nous apporter des éclairages, ils ne peuvent pas tout maîtriser dans cet organisme vivant qu'est une langue. Ils sont utiles mais n'oublions pas l'usage et le pragmatisme des locuteurs. Donner une confiance aveugle aux seuls "pros" me paraît (en économie, en linguistique ou autre) dangereux, anti-démocratique et moins efficace car ces "pros" sont souvent eux-mêmes orientés. Il est bon de les bousculer dans leurs certitudes" (Varlet4 mai 2012: Thriller)

Nous avons d'un côté "l'usage et le pragmatisme des locuteurs" contre un monde "dangereux, anti-démocratique et moins efficace" des "pros".

Quel est donc le sens que Varlet donne à l'aspect démocratique de l'usage populaire de la langue? Pourquoi le savoir spécialisé et professionnel en linguistique serait-il dangereux ? L'usage et le pragmatisme des locuteurs sont présentés ici comme un savoir "démocratique, non dangereux et efficace", ce qui pourrait faire écho à une autre intervention de thomas:

28. “ ... Car, c'est devenu clair que la Délégation Générale de langue française a crée ce site pour que nous les laissions tranquilles. Ils n'ont jamais pris une seule suggestion qu'on a proposée, ils ne cherchaient qu'à faire une foire à discussion, alors qu'ils s'efforcent de trouver des solutions très très intéressantes comme "pharmacovigiliance"... même "amarsissage" était interdit! Ce dernier avait le soutien du peuple, Le Monde, la logique... (thomas 1 octobre 2012)

Thomas revendique le droit à la prise en compte des néologismes des internautes, une sorte de légitimation du savoir du "peuple". Nous pouvons penser que la démocratie est définie d'abord comme le savoir non spécialisé sur la langue (chez Varlet) mais aussi comme reconnaissance de ce savoir par les instances institutionnelles (chez thomas). Nous nous arrêtons donc sur ce savoir des contributeurs, au sujet du métalangage, afin de mieux comprendre leurs manières de créer et de discuter de la langue, au-delà des savoirs étymologiques et des critères contextuels et quantitatifs.

\section{MÉTALANGAGE NON SPÉCIALISÉ}

Nous allons décrire d'un côté le type de métalangage utilisé par les internautes et d'un autre côté le rapport à la langue rendu manifeste par ce métalangage. Pour ce qui est du métalangage, les commentaires sur WikiLF font circuler un vocabulaire plutôt 
scolaire. Même s'ils montrent une bonne connaissance des notions, cela s'arrête à des domaines fort connus comme la phonétique, la sémantique et la morphologie.

Afin de créer un mot, les contributeurs se réfèrent souvent à la phonétique soit pour une francisation du mot à traduire :

29. “Le problème est que ce mot n'est pas francisé... Il ne s'adapte pas aux structures linguistiques du français. Moi, je serais assez partant pour revenir vers l'étymologie (Geck : "fou" en allemand) et d'utiliser sa forme la plus francisée : le picard "gicques" (tamdegledel 1 octobre 2011 : Geek)

Soit pour un usage lié à "une proximité phonétique" avec l'anglais :

30. "Il faut faire bref pour être écrit sur les ardoises des tenanciers de bistrot et compréhensible par tous aussi je propose "Apéro-heure" Apéro a de surcroît une proximité phonétique avec happy" (Marie 6 octobre 2011: Happy hour)

En morphologie, la notion de "dérivation" est introduite afin de remédier à un manque de métalangage - cependant sans visée corrective :

31. "Pour blogue, on dit CARNET. Suivant la méthodologie de journal > journaliste, je vais proposer CARNETTISTE" (homas 18 décembre 2011: Blogueur)

32. "Si on applique la même dérivation morphologique de Journal > Journaliste, on pourrait obtenir bloguiste à partir de blog" (Marou 17 juin 2012: Blogueur)

En sémantique, les notions sont plutôt scolaires; il est souvent question de "synonyme" et de "connotation" tous deux présents dans un même commentaire:

33. "De mon avis, en France, le terme "geek" est désormais synonyme de "fan d'informatique" ou "fan de nouvelles technologies". Donc si on reste dans cette traduction populaire, il faut exclure certains termes et ne pas oublier qu'il faudrait un terme qui ait une connotation informatique/nouvelles technologies" (Labbai 1 octobre 2011 Geek)

Ou bien la synonymie est évoquée dans le cadre de la règle scolaire de "ne pas se répéter":

34. "... Il est toujours utile d'avoir un synonyme pour, dans un discours, ne pas se repeater" (fosto 3 octobre 2011: Geek)

Si le métalangage ne semble pas sophistiqué, les contributeurs tendent à créer le leur, toujours en lien avec un vocabulaire linguistique, révélant alors un rapport "spontané" au métalangage. Anoup n'hésite pas à créer un registre de langue rarement utilisé dans les manuels de linguistique:

35. "Ambianceuse", experte en ambiances élégantes et festives [...] Par un retour savoureux, la principale confrérie d'ambianceurs s'appelle la "SAPE", Société des Ambianceurs et Personnes Elégantes, ce qui fait un jeu de mots avec l'argot français "sape", vêtement, souvent dans le registre élégant: "être sapé comme un milord" (Anoup 2 novembre 2011: Fashionitas)

En plus de l'invention d'un registre, nous avons des commentaires révélant un manque de souci d'exactitude terminologique: il importe peu à roze de parler de néologisme puisqu'elle a décidé de parler d'idiolecte :

36. “Au risque de créer un idiolecte, je dirais une tusse ;-)" (roze 28 novembre 2011 : Mug) 
À ce "risque", un autre locuteur réagit en essayant de rectifier "idiolecte" qui pour lui "n'existe pas":

37. “À Roze: bien pour idiolecte sauf que cette lexie n'existe pas: il s'agirait plutôt d'un idiotisme ou alors dans le sens que vous semblez lui donner, un néologisme" (phiral 3 décembre 2011)

Malgré cette rectification, roze réaffirme le côté idiolectal traduit, à sa façon, par singulier et exclusif :

38. "Oui Phiral, on peut l'appeler néologisme ce mot de "tusse", mais comme je n'ignore pas que sa diffusion est improbable, je le qualifiais d'idiolecte, puisqu'il demeurera tel. En somme, grâce à cet exercice, j'aurais au moins trouvé un mot qui me plaît et dont j'aurai sans doute l'usage exclusive" (roze 5 décembre 2011)

Idiolecte ou néologisme, la précision notionnelle ne paraît guère importante. Plus encore, certains contributeurs inventent des expressions construisant leur jargon:

39. “@ nOOn: nOOn! Même si Geek voulait dire a l'origine "fou du village" l'usage courant de ce lui a fait perdre de la force lexicale, Aujourd'hui le geek n'est que quelqu'un de plus passionné que les autres sur un sujet" (Eric_picture 28 septembre 2011 : Geek)

Ici "la force" est associé au lexique. Ailleurs nous trouvons l'expression "soif lexicale":

40. "Le terme (retour pédagogique) est bon et correct mais apparemment pas assez sexy puisque les locuteurs lui préfèrent feedback. Il faut satisfaire la "soif lexicale" des locuteurs" (Pixelboss 5 octobre 2011: feedback)

Sans s'arrêter sur ce que signifient la "force" et la "soif" lexicales, nous constatons un rapport spontané et inventif au métalangage, mais aussi à la langue, où le néologisme devrait être "sexy". Entre un vocabulaire peu spécialisé et une tendance à proférer leur propre métalangage, les contributeurs sur WikiLF jouissent d'une bonne connaissance de certaines notions grammaticales et linguistiques qui semblent assez générales, et partagées par linguistes et non linguistes.

\section{MISE EN DISCUSSION ET RAPPORT À LA LANGUE}

Notre analyse du métalangage utilisé sur WikiLF dans le contexte de création néologique aide à définir ce rapport des contributeurs moins comme des représentations sur la langue française que des règles de création langagière, en plus de ce que nous appelons une mise en discussion pacifique de la langue. Autrement dit, il s'agit de discuter les contraintes et critères de création néologique (tendances opposées et communes). Les locuteurs échangent des informations, corrigent certaines notions sans porter un jugement sur l'imprécision terminologique de leurs co-contributeurs ; ils répondent par là à l'objectif même de l'outil numérique qu'est un wiki. Aussi, il importe de préciser que la langue ne fait pas l'objet de dispute et que WikiLF demeure un espace de discussion où "passions et polemiques" n'ont pas de place. Les rares polémiques autour du métalangage n'ont pas pour objet la langue mais le vocabulaire des spécialistes, ce qui est intéressant à étudier d'autant plus que le savoir des linguistes a été qualifié d' "anti-démocratique" . La démocratie trouve alors sa place dans une discussion sur 
le savoir non spécialisé, confronté au savoir spécialisé. Nous nous arrêtons ici sur la réponse du locuteur à la polémique déjà signalée, en voici quelques éléments:

41.Varlet a stipulé: “En conclusion, je dirais que la langue est une affaire trop sérieuse pour être abandonnée aux seuls pseudo-linguistes, trop enfermés dans leurs raisonnements catégoristes et qu'elle est d'abord ce que les gens en font dans leur quotidien". Il a écrit également: "On aura beau se baser sur des études lexicales ou linguistiques pour inventer un autre mot..." 2) "pseudo-linguistes"? en vertu de quelle autorité scientifique le mot "pseudo" est-il utilisé? 4) "ce que les gens en font": les gens font-ils toujours le bien "dans leur quotidien"? Le fait que" "les gens" de Müllheim (Pays de Bade/Allemagne) aient voté pour Hitler à 63\% en 1933 rien que parce que le prix des fruits, des céréales et du vin s'était effondré, est-il du au fait que la langue d'Hitler était " d'abord ce que les gens en font" ? Le présent site n'aura des chances de survie et d'un tant soit peu de reconnaissance que moyennant un minimum de tolérance envers les "pros" de la linguistique, de la terminologie, des traducteurs, des interprètes, qui bien plus que l'on ne pense, s'occupent... des gens (Zinnkoepfle-Niadrbrennla 4 mai 2012).

Au-delà de la problématique du rapport à la langue, cette polémique sur le métalangage suscite trois questionnements: Le contributeur répond à la polémique en se demandant si "les gens font toujours le bien dans leur quotidien". Il semble suggérer qu'il existe des connaissances des gens contre des connaissances spécialisées des professionnels des langues et des linguistes.

Les connaissances des gens sont ici pointées comme étant incomplètes voire dangereuses puisque Zinnkoepfle-Niadrbrennla assimile ce savoir au fait que "les gens" ont pu voter pour Hitler. La question de l'incomplétude du savoir non spécialisé, ou "des gens", est en effet à poser, or ce locuteur semble juger ces connaissances, qui ailleurs dans le domaine des sciences du langage et des linguistes qu'il défend, font l'objet d'amples études et travaux en linguistique sur "la linguistique des non linguistes" ou ce qui est appelé "la linguistique populaire" et ailleurs " folk linguistics" (entre autres ACHARD-BAYLE, 2008, NIEDZIELSKI; PRESTON, 2000). Nous notons ici une "attitude plutôt polemique" de la part du locuteur divisant le monde des savoirs en deux et ignorant que les connaissances "des gens" constituent un savoir à part entier mais aussi un champ d'étude des linguistes eux-mêmes.

L'argument de "Hitler" semble excessif, le contributeur bloque la discussion par "la Loi Godwin" qui se traduit dans les termes suivants: "Plus une discussion en ligne dure longtemps, plus la probabilité d'y trouver une comparaison impliquant les nazis s'approche de 1". ${ }^{160}$ Ce "point Godwin" est une invention d'Internet signalant ainsi l'échec de la discussion. Certes, il serait excessif voire illogique de penser que le savoir des linguistes est "anti-démocratique", néanmoins il conviendrait peut-être d'expliquer plus clairement comment la linguistique peut être une science utile qui répond à des questions de société au lieu d'aller dans le sens de jugements de valeurs contre "les connaissances des gens".

Le contributeur se trouve dans la situation de défense où il revendique une forme de tolérance vis-à-vis des linguistes. L'on se demande si le fait que WikiLF permette,

160 Voir Brogol (2010). 
de fait, une confrontation entre spécialistes et non spécialistes, ne pourrait le faire glisser vers un espace d'opinions confuses, de la part des non linguistes qui peuvent "devenir linguistes", et de la part des linguistes qui deviennent des "vendeurs/défenseurs de leur savoir". Ces deux situations impliquent une confusion dans les rôles de chacun au sens de ce que les uns peuvent apporter aux autres.

Aux termes de ces questionnements, nous considérons qu'il n'est pas anodin de voir émerger le mot "démocratie" dans un wiki de création néologique. Il serait intéressant de réfléchir sur ce que recouvre ce terme et ce qu'il dit sur les rapports entre savoir non spécialisé et spécialisé à l'heure du numérique, lequel a créé plusieurs amalgames, par exemple entre "bloggeur" et "auteur", entre "amateur" et "professionnel" (MANSOUR; RINCK, 2013). C'est en effet la question auctoriale qui est là pointée : le web a mis à disposition des outils et des plateformes (comme les wikis) permettant à la créativité de chacun de trouver sa place, ce qui fait l'objet de débats et d'études sur une éventuelle confusion entre des notions "d'auteur" et "d'amateur" (en domaine artistique entre autres), et dans notre cas ici cela pose la question des limites qu'il existe entre " un professionnel de la langue et/ou linguiste" et "un non linguiste non professionnel de la langue". Il conviendrait de réfléchir à l'avenir sur les effets et l'impact du web dans la création tout comme dans l'effacement des frontières entre experts, savants et connaissances dites populaires. Il ne s'agirait pas de créer ces frontières ni de les effacer mais de les repenser, étudiant plutôt un aller-retour entre différents modes de production du savoir.

Pour finir, cette revendication d'une reconnaissance chez le profane n'aurait peutêtre pas eu lieu s'il n'avait pas été question du métalangage. Le métalangage semble le lieu des limites d'un savoir populaire, même si les linguistes peuvent aussi discuter de leur terminologie (GLIKMAN; MANSOUR; WEISER, 2011). Au-delà de la linguistique et des études sur la langue et face à tout jargon professionnel, tout locuteur peut sembler dépourvu de moyens de compréhension. Une certaine "peur" peut se manifester à l'écoute de "notions" et de toute terminologie spécialisée, à l'instar des situations d'incompréhension devant une prescription médicale. Ainsi, le métalangage des linguistes tout comme le jargon professionnel de tout métier n'est pas épargné de l'étiquette "langue étrangère" voire "charabia" pour certains. Notre objet d'étude choisi qui est là l'usage par les non linguistes/non spécialistes du métalangage lors de création de nouveaux mots pourrait expliquer ce rejet qui a été exprimé face à des "catégorisations" enfermantes.

Du côté des linguistes, la réflexion sur le métalangage constitue un travail de longue haleine sur leurs objets scientifiques (entre autres GLIKMAN; MANSOUR; WEISER 2010). Nous sommes alors loin du métalangage des wikistes qui, au détour d'un commentaire et le temps d'une minute, peuvent jongler avec le vocabulaire spécialisé ; se donnant ainsi plus de marge de manoeuvre dans leurs échanges, et plus de liberté à réinventer la langue à chaque seconde, quand un linguiste passe des années à comprendre son fonctionnement et son usage.

À la fin de cette réflexion sur l'usage du métalangage sur Wikilf, nous pouvons mieux définir le rapport spontané des locuteurs francophones à leur langue qui semble ici dénué de toute passion, éloigné des postures normatives. Les wikistes donnent l'exemple d'une communauté qui pacifiquement met en discussion la langue 
française, comme un contre-discours normatif lequel se définit par un contre exemple des études sur le rapport puriste et normatif à la langue. Ce qui représente pour nous un objet de réflexion, de discussion, mais aussi un choix de recherche permettant de nous positionner afin de rendre compte (et de prendre en compte) d'une relation plus souple sinon assouplie des usagers de la langue française. 


\section{BIBLIOGRAPHIE}

ACHARD-BAYLE, G. La linguistique "hors du temple", Introduction la Pratiques. 139/140, “Linguistique populaire?”, pp. 3-16, 2008.

BROGOL. En finir avec la loi Godwin. La Politeia, 2010. En ligne sur: <http://lapoliteia.com/ en-finir-avec-le-point-godwin-critique-de-la-loi-de-godwin/>. Consulté le: 5 sept. 2013.

CERQUIGLINI, B. Une langue orpheline. Paris: Éditions du Minuit, 2007

CHARAUDAU, P.; MAINGUENEAU, D. Dictionnaire d'analyse du discours. Paris: Seuil, 2002.

DIETMAR, O. Le bon usage d'internet. Le discours normatif sur la toile, 2004. En ligne sur: <http://www.dietmar-osthus.de/norme.htm>.

FOUCAULT, M. L'archéologie du savoir. Paris: Gallimard, 1969.

GLIKMAN, J., MANSOUR, L. \&WEISER, S. (Éds). Le vocabulaire technique et scientifiques en sciences du langage. Actes de col'doc 2007, 2010. En ligne sur: 〈www.modyco.fr〉.

MANSOUR, L.; RINCK, F. La littératie à l'ère numérique: le copier-coller chez les étudiants . Linguagem em (dis)curso. Coord. F. Komesu et L. Tenani, Brésil, 2013.

MESCHONNIC, H. De la langue française. Paris: Hachette Littératures, 1997.

NIEDZIELSKI, N; PRESTON, D. Folk linguistics. Berlin, New York: Mouton de Gruyter, 2000.

PAVEAU, M.-A. Du contexte à l'environnement: pour une approche écologique du discours, conférence donnée in La langue en contexte, JE DosCila Paris Diderot, 2013.

; ROSIER, L. La langue française. Passions et polémiques. Paris: Vuibert, 2008.

ROSIER, L. La circulation des discours à la lumière de "l'effacement énonciatif": l'exemple du discours puriste sur la langue . Langages 156, pp. 65-78, 2004.

STEUCKARDT, A. Néologie et sentiment de la langue française au xviiie siècle. Diachroniques 2, pp. 81-101, 2012. 